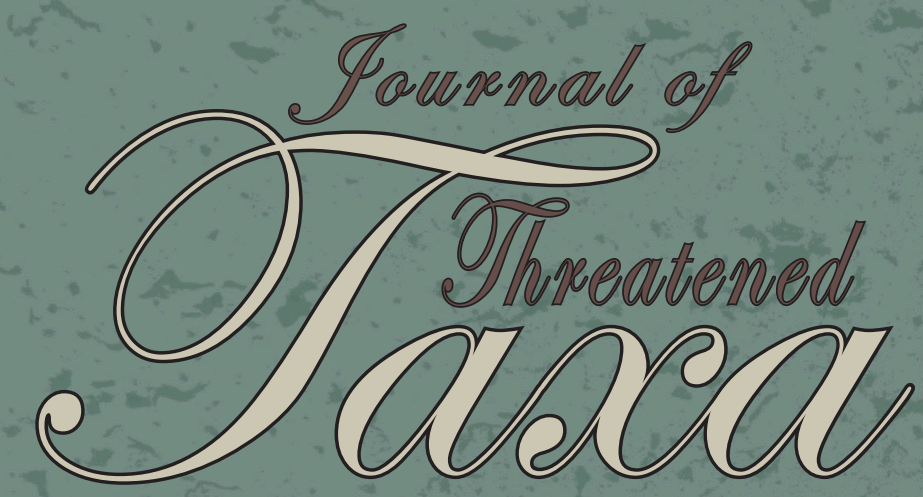

Building evidence for conservation globally
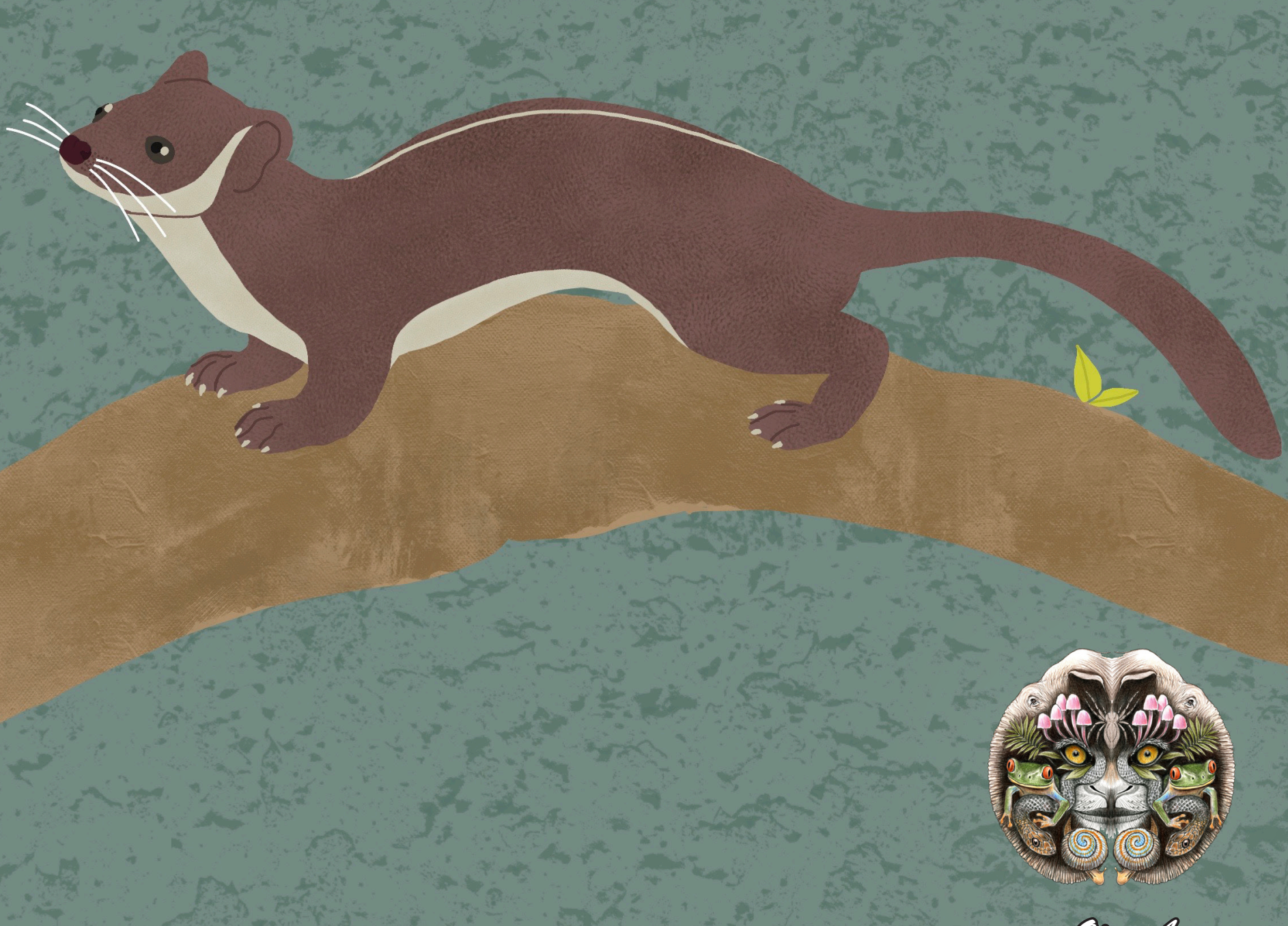

Qpecosecess

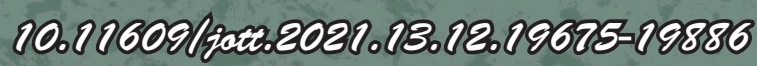
cocosurthreatecredtassararg

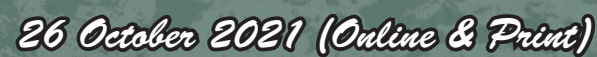

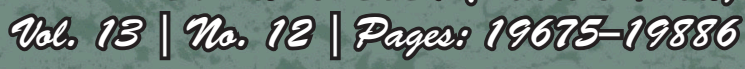




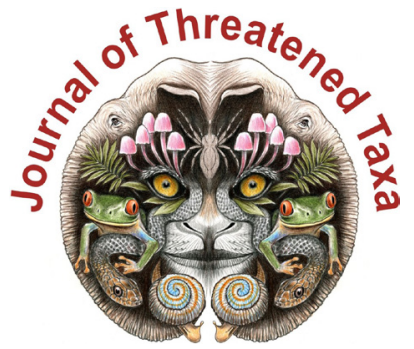

ISSN 0974-7907 (Online); ISSN $0974-7893$ (Print)

Publisher

Host

Wildlife Information Liaison Development Society

www.wild.zooreach.org

Zoo Outreach Organization www.zooreach.org

No. 12, Thiruvannamalai Nagar, Saravanampatti - Kalapatti Road, Saravanampatti, Coimbatore, Tamil Nadu 641035, India

Ph: +91 9385339863 | www.threatenedtaxa.org

Email: sanjay@threatenedtaxa.org

EDITORS

\section{Founder \& Chief Editor}

Dr. Sanjay Molur

Wildlife Information Liaison Development (WILD) Society \& Zoo Outreach Organization (ZOO)

12 Thiruvannamalai Nagar, Saravanampatti, Coimbatore, Tamil Nadu 641035, India

\section{Deputy Chief Edito}

Dr. Neelesh Dahanukar

Noida, Uttar Pradesh, India

\section{Managing Editor}

Mr. B. Ravichandran, WILD/ZOO, Coimbatore, India

\section{Associate Editors}

Dr. Mandar Paingankar, Government Science College Gadchiroli, Maharashtra 442605, India Dr. Ulrike Streicher, Wildlife Veterinarian, Eugene, Oregon, USA

Ms. Priyanka Iyer, ZOO/WILD, Coimbatore, Tamil Nadu 641035, India

Dr. B. A. Daniel, $200 /$ WIID, Coimbatore, Tamil Nadu 641035, India

\section{Editorial Board}

Dr. Russel Mittermeie

Executive Vice Chair, Conservation International, Arlington, Virginia 22202, USA

\section{Prof. Mewa Singh Ph.D., FASc, FNA, FNASc, FNAPsy}

Ramanna Fellow and Life-Long Distinguished Professor, Biopsychology Laboratory, and Institute of Excellence, University of Mysore, Mysuru, Karnataka 570006, India; Honorary Professor, Jawaharlal Nehru Centre for Advanced Scientific Research, Bangalore; and Adjunct Professor, National Institute of Advanced Studies, Bangalore

\section{Stephen D. Nash}

Scientific Illustrator, Conservation International, Dept. of Anatomical Sciences, Health Sciences Center, T-8, Room 045, Stony Brook University, Stony Brook, NY 11794-8081, USA

\section{Dr. Fred Pluthero}

Toronto, Canada

\section{Dr. Priya Davidar}

Sigur Nature Trust, Chadapatti, Mavinhalla PO, Nilgiris, Tamil Nadu 643223, India

\section{Dr. Martin Fisher}

Senior Associate Professor, Battcock Centre for Experimental Astrophysics, Cavendish

Laboratory, JJ Thomson Avenue, Cambridge CB3 OHE, UK

\section{Dr. John Fellowes}

Honorary Assistant Professor, The Kadoorie Institute, 8/F, T.T. Tsui Building, The University of Hong Kong, Pokfulam Road, Hong Kong

\section{Prof. Dr. Mirco Solé}

Universidade Estadual de Santa Cruz, Departamento de Ciências Biológicas, Vice-coordenado do Programa de Pós-Graduação em Zoologia, Rodovia Ilhéus/Itabuna, Km 16 (45662-000)

Salobrinho, Ilhéus - Bahia - Brasil

\section{Dr. Rajeev Raghavan}

Professor of Taxonomy, Kerala University of Fisheries \& Ocean Studies, Kochi, Kerala, India

\section{English Editors}

Mrs. Mira Bhojwani, Pune, India

Dr. Fred Pluthero, Toronto, Canad

Mr. P. Ilangovan, Chennai, India

Web Maintenance

Mrs. Latha G. Ravikumar, ZOO/WILD, Coimbatore, India

\section{Typesetting}

Mr. Arul Jagadish, ZOO, Coimbatore, India

Mrs. Radhika, ZOO, Coimbatore, India

Mrs. Geetha, ZOO, Coimbatore India

\section{Fundraising/Communications}

Mrs. Payal B. Molur, Coimbatore, India

Subject Editors 2018-2020

Fungi

Dr. B. Shivaraju, Bengaluru, Karnataka, India

Dr. R.K. Verma, Tropical Forest Research Institute, Jabalpur, India

Dr. Vatsavaya S. Raju, Kakatiay University, Warangal, Andhra Pradesh, India

Dr. M. Krishnappa, Jnana Sahyadri, Kuvempu University, Shimoga, Karnataka, India

Dr. K.R. Sridhar, Mangalore University, Mangalagangotri, Mangalore, Karnataka, India

Dr. Gunjan Biswas, Vidyasagar University, Midnapore, West Bengal, India

Plants

Dr. G.P. Sinha, Botanical Survey of India, Allahabad, India

Dr. N.P. Balakrishnan, Ret. Joint Director, BSI, Coimbatore, India

Dr. Shonil Bhagwat, Open University and University of Oxford, UK

Prof. D.J. Bhat, Retd. Professor, Goa University, Goa, India

Dr. Ferdinando Boero, Università del Salento, Lecce, Italy

Dr. Dale R. Calder, Royal Ontaro Museum, Toronto, Ontario, Canada

Dr. Cleofas Cervancia, Univ. of Philippines Los Baños College Laguna, Philippines

Dr. F.B. Vincent Florens, University of Mauritius, Mauritius

Dr. Merlin Franco, Curtin University, Malaysia

Dr. V. Irudayaraj, St. Xavier's College, Palayamkottai, Tamil Nadu, India

Dr. B.S. Kholia, Botanical Survey of India, Gangtok, Sikkim, India

Dr. Pankaj Kumar, Kadoorie Farm and Botanic Garden Corporation, Hong Kong S.A.R., China

Dr. V. Sampath Kumar, Botanical Survey of India, Howrah, West Bengal, India

Dr. A.J. Solomon Raju, Andhra University, Visakhapatnam, India

Dr. Vijayasankar Raman, University of Mississippi, USA

Dr. B. Ravi Prasad Rao, Sri Krishnadevaraya University, Anantpur, India

Dr. K. Ravikumar, FRLHT, Bengaluru, Karnataka, India

Dr. Aparna Watve, Pune, Maharashtra, India

Dr. Qiang Liu, Xishuangbanna Tropical Botanical Garden, Yunnan, China

Dr. Noor Azhar Mohamed Shazili, Universiti Malaysia Terengganu, Kuala Terengganu, Malaysia Dr. M.K. Vasudeva Rao, Shiv Ranjani Housing Society, Pune, Maharashtra, India

Prof. A.J. Solomon Raju, Andhra University, Visakhapatnam, India

Dr. Mandar Datar, Agharkar Research Institute, Pune, Maharashtra, India

Dr. M.K. Janarthanam, Goa University, Goa, India

Dr. K. Karthigeyan, Botanical Survey of India, India

Dr. Errol Vela, University of Montpellier, Montpellier, France

Dr. P. Lakshminarasimhan, Botanical Survey of India, Howrah, India

Dr. Larry R. Noblick, Montgomery Botanical Center, Miami, USA

Dr. K. Haridasan, Pallavur, Palakkad District, Kerala, India

Dr. Analinda Manila-Fajard, University of the Philippines Los Banos, Laguna, Philippines

Dr. P.A. Sinu, Central University of Kerala, Kasaragod, Kerala, India

Dr. Afroz Alam, Banasthali Vidyapith (accredited A grade by NAAC), Rajasthan, India

Dr. K.P. Rajesh, Zamorin's Guruvayurappan College, GA College PO, Kozhikode, Kerala, India

Dr. David E. Boufford, Harvard University Herbaria, Cambridge, MA 02138-2020, USA

Dr. Ritesh Kumar Choudhary, Agharkar Research Institute, Pune, Maharashtra, India

Dr. Navendu Page, Wildlife Institute of India, Chandrabani, Dehradun, Uttarakhand, India

Invertebrates

Dr. R.K. Avasthi, Rohtak University, Haryana, India

Dr. D.B. Bastawade, Maharashtra, India

Dr. Partha Pratim Bhattacharjee, Tripura University, Suryamaninagar, India

Dr. Kailash Chandra, Zoological Survey of India, Jabalpur, Madhya Pradesh, India

Dr. Ansie Dippenaar-Schoeman, University of Pretoria, Queenswood, South Africa

Dr. Rory Dow, National Museum of natural History Naturalis, The Netherlands

Dr. Brian Fisher, California Academy of Sciences, USA

Dr. Richard Gallon, llandudno, North Wales, LL30 1UP

Dr. Hemant V. Ghate, Modern College, Pune, India

Dr. M. Monwar Hossain, Jahangirnagar University, Dhaka, Bangladesh

Mr. Jatishwor Singh Irungbam, Biology Centre CAS, Branišovská, Czech Republic.

Dr. lan J. Kitching, Natural History Museum, Cromwell Road, UK

Dr. George Mathew, Kerala Forest Research Institute, Peechi, India

Dr. John Noyes, Natural History Museum, London, UK

For Focus, Scope, Aims, and Policies, visit https://threatenedtaxa.org/index.php/JoTT/aims_scope
For Article Submission Guidelines, visit https://threatenedtaxa.org/index.php/JoTT/about/submissions
For Policies against Scientific Misconduct, visit https://threatenedtaxa.org/index.php/JoTT/policies_various

continued on the back inside cover 


\title{
Additional distribution records of Ceropegia anjanerica, an endemic and 'Endangered' lantern flower of the northern Western Ghats, India
}

\author{
Samir Shrikant Maity ${ }^{1} \mathbb{D}$, Ajay Natha Gangurde ${ }^{2} \mathbb{D}$, Sharad Suresh Kambale ${ }^{3} \mathbb{D}$, \\ Avinash Ramchandra Gholave ${ }^{4} \mathbb{D}$, Avinash Asraji Adsul ${ }^{5} \mathbb{D}$, Ganesh Babaso Pawar ${ }^{6} \mathbb{D}$ \& \\ Kumar Vinod Chhotupuri Gosavi ${ }^{7}$ (i)
}

\footnotetext{
1,7 Department of Botany, Gokhale Education Society's HPT Arts and RYK Science College, Nashik, Maharashtra 422005, India.

${ }^{2}$ Department of Botany, Savitribai Phule Pune University, Pune, Maharashtra 411007, India.

${ }^{3}$ Department of Botany, Maratha Vidya Prasarak Samaj's Arts, Commerce \& Science College, Tryambakeshwar, Nashik, Maharashtra 422212, India.

${ }^{4}$ Department of Botany, K.V.N. Naik Arts, Commerce and Science College, Canada Corner, Nashik, Maharashtra 422002, India. ${ }^{5}$ Department of Botany, Gokhale Education Society's Arts, Commerce and Science College, Jawhar, District Palghar,
} Maharashtra 401603, India.

${ }_{6}^{6}$ Department of Botany, Abasaheb Marathe Arts and New Commerce, Science College, Rajapur, District-Ratnagiri, Maharashtra 416702, India. ${ }^{1}$ samirmaity6888@gmail.com, ${ }^{2}$ ajaygangurde98@gmail.com, ${ }^{3}$ skambalesu@gmail.com (corresponding author), ${ }^{4}$ agholave@gmail.com, 5avinash.adsul@gmail.com, ${ }^{6}$ pawargb22@gmail.com, ${ }^{7}$ kumarvinodgosavi@gmail.com

Genus Ceropegia L. (s.s.) (Ceropegiae: Apocynaceae) is represented by more than 260 taxa worldwide (The Plant List 2013) and distributed in the Canary Islands, India, Madagascar, New Guinea, northern Australia, southeastern Asia, tropical Arabica, and Africa except the Mediterranean region (Meve 2002). It is represented by 62 taxa in India and 26 taxa in Maharashtra (Kambale \& Yadav 2019; Murugesan et al. 2019). Nashik district represents six species and two varieties of Ceropegia, viz., Ceropegia anjanerica Malpure, M.Y.Kamble \& S.R.Yadav, C. bulbosa Roxb. var. bulbosa, C. bulbosa Roxb. var. lushii (Graham) Hook.f., C. hirsuta Wight \& Arn., C. lawii Hook.f., C. mahabalei Hemadri \& Ansari, C. media (H.Huber) Ansari, and C. vincifolia Hook (List modified based on personal observations, after
Lakshminarasimhan \& Sharma 1991).

Ceropegia anjanerica is an 'Endangered' (Pethe \& Watve 2021) and endemic flytrap flower and has recently been reported from adjacent area called Navardev, Kushegaon, Igatpuri tehsil of Nashik district (Auti et al. 2019).

As a part of floristic studies on Tryambakeshwar and surrounding areas, various places have been surveyed by the authors. A recent survey made of the Bhaskargad, Bramha Hill, Bramhagiri Hill, Harihar Fort, Vatvad Hill, (Nashik District) resulted in the collection of Ceropegia anjanerica (Malpure et al. 2006).

Ceropegia anjanerica Malpure, M.Y.Kamble \& S.R. Yadav Curr. Sci. 91(9): 1141. 2006; Karthik. et al. FI. PI.

Citation: Maity, S.S., A.N. Gangurde, S.S. Kambale, A.R. Gholave, A.A. Adsul, G.B. Pawar \& K.V.C. Gosavl (2021). Additional distribution records of Ceropegia anjanerica, an endemic and 'Endangered' lantern flower of the northern Western Ghats, India. Journal of Threatened Taxa 13(12): 19874-19877. https://doi. org/10.11609/jott.6879.13.12.19874-19877

Copyright: ( Maity et al. 2021. Creative Commons Attribution 4.0 International License. JoTT allows unrestricted use, reproduction, and distribution of this article in any medium by providing adequate credit to the author(s) and the source of publication.

Funding: Self-funded.

Competing interests: The authors declare no competing interests.
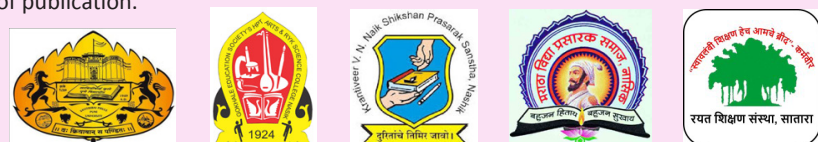

Acknowledgements: The authors thank the principals of their respective colleges for necessary facilities; Dr. Amol Mamlaya, Arts, Commerce and Science College, Mokhada for help during field work; Mr. Pankaj Garg (DCF), Mrs. Ratna Tuplondhe, Mr. Sujit Bokade, Mr. Dnyaneshwar Shinde, Mr. Haribhau Nimbekar, Mr. Sanjay Badade, Mr. Kailas Nimbekar \& Mr. Bablu Dive (Forest guards, Maharashtra Forest Department, Nashik West) for necessary help; and Mr. Rangnath Burange for information on the locality details. 

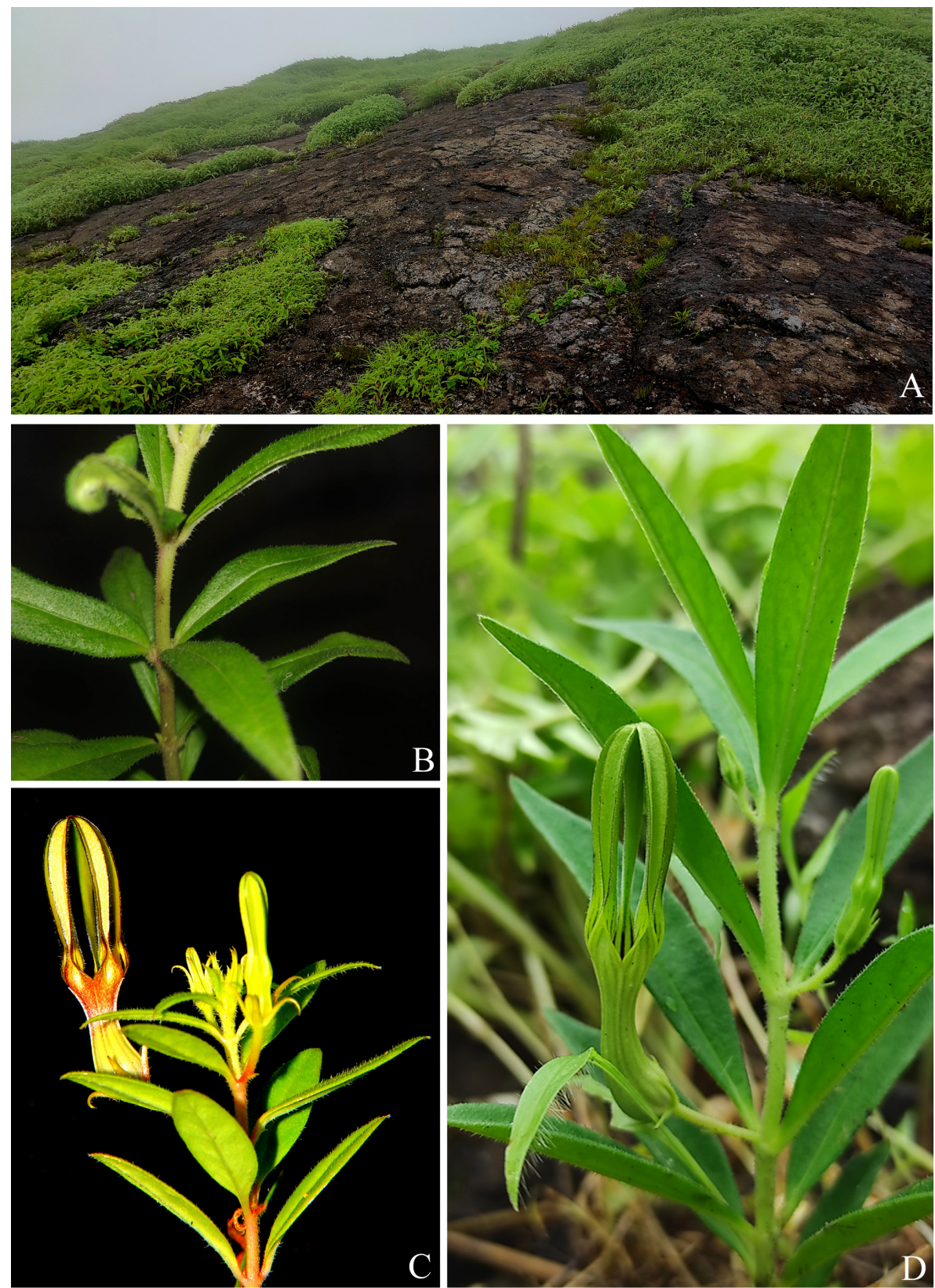

Image 1. Ceropegia anjanerica Malpure, M.Y. Kamble \& S.R. Yadav: A-Habitat (Bhaskargad) | B-Whorled leaves (Bramhagiri) | C-Kushegaon Population | D-Vatvad population. @ S.S. Maity.

India 1: 160. 2009; Kambale \& S.R.Yadav, Asklepios 115: 29. 2013; Kambale \& S.R.Yadav, Rheedea 29(1): 93; Auti et al. J. Bombay Nat. Hist. Soc. 116. 181. 2019. (Fig. 1, Table 1).

Perennial, erect, tuberous herbs. Stems rarely two per tuber. Leaves scabrous above, along midrib, and margin, lamina lamina elliptic-narrowly elliptic, 1.3-3.7 $\times 0.3-1.1 \mathrm{~cm}$, glabrous otherwise. Inflorescence an extra-axillary solitary flower; corolla tube up to $1.9 \mathrm{~cm}$ long, gradually dilated at base, funnel-shaped towards throat, slightly curved, greenish-grey, striated with deep purple lines within, white otherwise, glabrous within; corolla lobes $1.4-1.9 \mathrm{~cm}$ long, greenish-yellow, obovate, attenuate, finely pubescent throughout, connate at the tip, lobes reflexed. Corona biseriate, stipitate; outer corona of five bifid lobes, $2 \times 2 \mathrm{~mm}$, yellow, ciliate within and along margin; inner corona of five erect linear lobes, c. $2 \mathrm{~mm}$ long, alternating with outer corona. Follicles usually in pairs, straight, tapering to a fine point, erect. Seeds ovoid, oblong, comose; coma white, silky.

Flowering \& Fruiting: July-November.

Distribution: Endemic to the northern Western Ghats 
Table 1. Comparison of characters at different populations.

\begin{tabular}{|l|l|l|l|l|}
\hline Character & Anjaneri & Bramhagiri & Harihar fort & Kushegaon \\
\hline Arrangement of leaves & opposite-decussate & whorled (Image 1B) & opposite-decussate & opposite-decussate \\
\hline Lamina shape & elliptic-narrowly elliptic & elliptic-narrowly elliptic & linear to lanceolate & elliptic-narrowly elliptic \\
\hline Corolla tube & green & green & green & red at the upper half (Image 1C) \\
\hline Corolla lobes & Yellowish-green & Yellowish-green & Yellowish-green & yellow with reddish tinge (Image 1C) \\
\hline
\end{tabular}

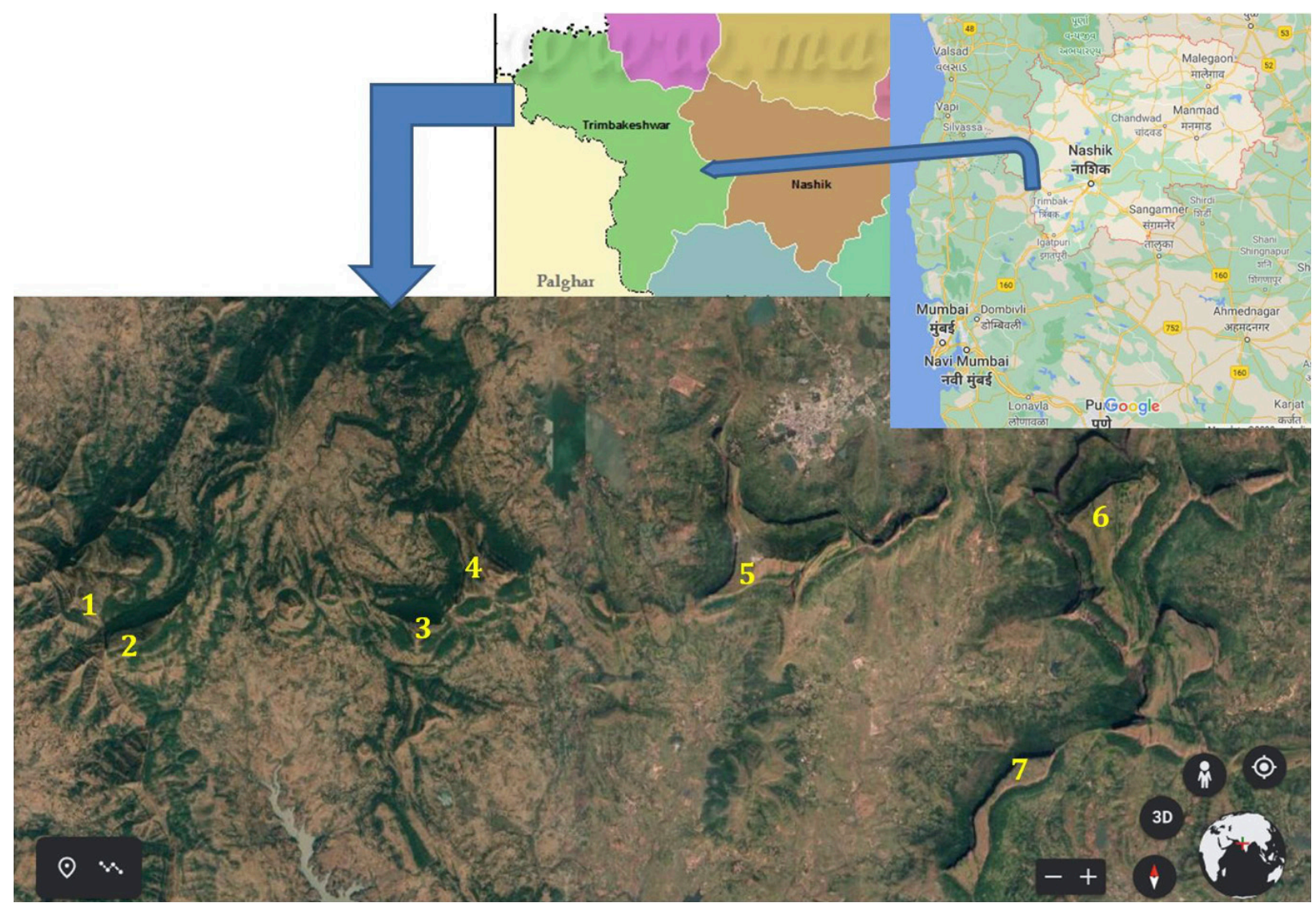

Image 2. Distribution of Ceropegia anjanerica Malpure, M.Y. Kambale \& S.R. Yadav: 1-Vatvad Hill | 2-Bhaskargad | 3-Harihar fort | 4Bramha Hill | 5-Bramhagiri Hill | 6-Anjaneri Hill (Type locality) | 7-Kushegaon.

of Maharashtra (Nashik district).

Habitat: Grows at an altitude of about 1,300 m in welldrained soil, in association with Cyanotis fasciculata (B. Heyne ex Roth) Schult.f., Justicia procumbens L., Senecio bombayensis N.P. Balakr. and Swertia minor Knobl.

Specimens examined: SSK-5420, 19.vii.2020, India, Maharashtra, Nashik District, Tryambakeshwar, Umbhrande, Vatvad Hill, coll. S.S. Kambale (Image 3); SSK-5421, 28.vii.2020, Bhaskargad, coll. A.N. Gangurde \& S.S. Maity; SSK-5422, 3.viii.2020, Harshvadi, Harihar Fort, coll. S.S. Maity \& A.N. Gangurde; SSK-5423, 4.viii.2020, Tryambakeshwar, Bramhagiri Hill, coll. S.S. Maity \& A.N. Gangurde; SSK-5427, 6.ix.2020, Bramha
Hill, Harshvadi-Talegaon, coll. A.N. Gangurde, S.S. Maity, A.A. Adsul \& S.S. Kambale (All specimens are in the Herbarium of Department of Botany, Arts, Commerce \& Science College, Tryambakeshwar).

Notes: These peculiar flytrap flowers remained unnoticed despite the localities of their occurrence were frequently visited by both botanists and amateur plant photographers. Vatvad Hill, Bhaskargad, Harihar Fort, and Brahmagiri are the places of the public interest and visited for adventurous treks during and after monsoon. Authors have visited Bramhagiri Hill several times, however, never encountered with Ceropegia anjanerica. This year when we visited Vatvad Hill during 


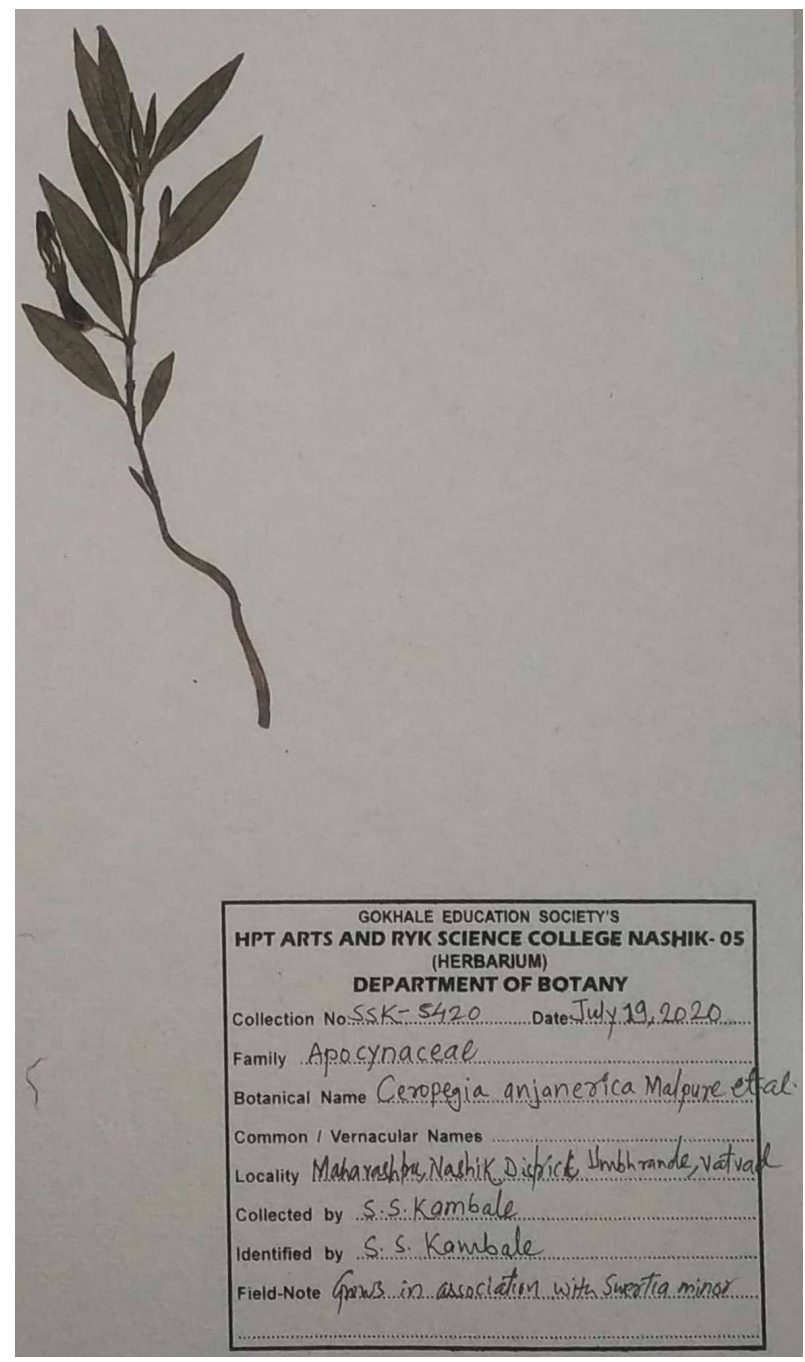

Image 3. Herbarium sheet of Ceropegia anjanerica (\# SSK-5420).

monsoon (July 2020) we observed healthy population of Ceropegia anjanerica. Then, we thought that the species may occur wherever similar habitats are available. Such similar habitats are available on the very next rock outcrops which are Bhaskargad, Harihar fort, Bramha Hill and Bramhagiri Hill. Surveys undertaken to these places resulted in the collection of Ceropegia anjanerica. This collection highlights the need of designated surveys to locate such endemic species which are reported from their type localities only. This will help in prioritizing the conservation of threatened species.
Conservation status: Ceropegia anjanerica was assessed as Critically Endangered [CR B1ab (iii,v) + B2 ab (iii)] by Pethe et al. (2015) and as 'Endangered' by Pethe \& Watve (2021) based on additional four locations on the IUCN Red List. Though the type locality, i.e., Anjaneri Hills, Nashik, is declared as an 'Anjaneri Conservation Reserve' and due care has been taken by Maharashtra Forest Department for its conservation, other localities are under constant anthropogenic pressure. Other than Anjaneri Conservation Reserve, all the localities are tourist places and therefore, frequent trampling by tourists will certainly destroy the habitats. Grazing is not a severe threat at the above mentioned localities. Controlled tourism and awareness amongst tourists will help in reducing the threat to some extent. Unnecessary uprooting of the tuber just for the sake of growing this endemic species in captivity should be avoided as it will not survive outside its habitat more than a year or so if appropriate care is not taken.

\section{References}

Auti, S.G., K.V.C. Gosavi, S. Golait \& S.S. Kambale (2019). Notes on Ceropegia anjanerica (Family: Apocynaceae): a critically endangered and endemic 'fly trap flower' of northern Western Ghats. Journal of the Bombay Natural History Society 116: 181-182.

Kambale, S.S. \& S.R. Yadav (2019). Taxonomic revision of Ceropegia (Apocynaceae: Ceropegieae) in India. Rheedea 29(1): 1-115.

Lakshminarasimhan, P. \& B.D. Sharma (1991). Flora of Nashik District. Botanical Survey of India, Calcutta, 644pp.

Malpure, N.V., M.Y. Kamble \& S.R. Yadav (2006). A new species of Ceropegia L. (Asclepiadaceae) from Western Ghats of India with a note on series Attenuatae Huber. Current Science 91(9): 1140-1142.

Meve, U. (2002). Ceropegia, pp. 63-106. In: Albers, F. \& U. Meve (eds). Illustrated Handbook of Succulent Plants: Asclepiadaceae. Springer, Berlin.

Murugesan, M., A.A. Mao, L.R. Meitei \& S.S. Kambale (2019) Ceropegia khasiana (Apocynaceae: Ceropegieae), a new species from Meghalaya, Northeast India. Gardens' Bulletin Singapore 71(2): 519-525.

Pethe, J., A. Tillu \& A. Watve (2015). Threat status assessment of Ceropegia anjanerica Malpure et al. (Magnoliopsida: Gentianales: Apocynaceae) from Anjaneri Hills, Nashik District, Maharashtra, India. Journal of Threatened Taxa 7(3): 6965-6971. https://doi. org/10.11609/JoTT.03772.6965-71

Pethe, J. \& A. Watve (2021). Ceropegia anjanerica. The IUCN Red List of Threatened Species 2021: e.T177964188A177969117. Downloaded on 10 October 2021. https://doi.org/10.2305/IUCN.UK.2021-2.RLTS. T177964188A177969117.en

The Plant List (2013). Version 1.1. http://www.theplantlist.org/ Accessed July 30, 2020. 
Dr. Albert G. Orr, Griffith University, Nathan, Australia

Dr. Sameer Padhye, Katholieke Universiteit Leuven, Belgium

Dr. Nancy van der Poorten, Toronto, Canada

Dr. Kareen Schnabel, NIWA, Wellington, New Zealand

Dr. R.M. Sharma, (Retd.) Scientist, Zoological Survey of India, Pune, India

Dr. Manju Siliwal, WILD, Coimbatore, Tamil Nadu, India

Dr. G.P. Sinha, Botanical Survey of India, Allahabad, India

Dr. K.A. Subramanian, Zoological Survey of India, New Alipore, Kolkata, India

Dr. P.M. Sureshan, Zoological Survey of India, Kozhikode, Kerala, India

Dr. R. Varatharajan, Manipur University, Imphal, Manipur, India

Dr. Eduard Vives, Museu de Ciències Naturals de Barcelona, Terrassa, Spain

Dr. James Young, Hong Kong Lepidopterists' Society, Hong Kong

Dr. R. Sundararaj, Institute of Wood Science \& Technology, Bengaluru, India

Dr. M. Nithyanandan, Environmental Department, La Ala Al Kuwait Real Estate. Co. K.S.C., Kuwait

Dr. Himender Bharti, Punjabi University, Punjab, India

Mr. Purnendu Roy, London, UK

Dr. Saito Motoki, The Butterfly Society of Japan, Tokyo, Japan

Dr. Sanjay Sondhi, TITLI TRUST, Kalpavriksh, Dehradun, India

Dr. Nguyen Thi Phuong Lien, Vietnam Academy of Science and Technology, Hanoi, Vietnam

Dr. Nitin Kulkarni, Tropical Research Institute, Jabalpur, India

Dr. Robin Wen Jiang Ngiam, National Parks Board, Singapore

Dr. Lional Monod, Natural History Museum of Geneva, Genève, Switzerland.

Dr. Asheesh Shivam, Nehru Gram Bharti University, Allahabad, India

Dr. Rosana Moreira da Rocha, Universidade Federal do Paraná, Curitiba, Brasil

Dr. Kurt R. Arnold, North Dakota State University, Saxony, Germany

Dr. James M. Carpenter, American Museum of Natural History, New York, USA

Dr. David M. Claborn, Missouri State University, Springfield, USA

Dr. Kareen Schnabel, Marine Biologist, Wellington, New Zealand

Dr. Amazonas Chagas Júnior, Universidade Federal de Mato Grosso, Cuiabá, Brasil

Mr. Monsoon Jyoti Gogoi, Assam University, Silchar, Assam, India

Dr. Heo Chong Chin, Universiti Teknologi MARA (UiTM), Selangor, Malaysia

Dr. R.J. Shiel, University of Adelaide, SA 5005, Australia

Dr. Siddharth Kulkarni, The George Washington University, Washington, USA

Dr. Priyadarsanan Dharma Rajan, ATREE, Bengaluru, India

Dr. Phil Alderslade, CSIRO Marine And Atmospheric Research, Hobart, Australia

Dr. John E.N. Veron, Coral Reef Research, Townsville, Australia

Dr. Daniel Whitmore, State Museum of Natural History Stuttgart, Rosenstein, Germany.

Dr. Yu-Feng Hsu, National Taiwan Normal University, Taipei City, Taiwan

Dr. Keith V. Wolfe, Antioch, California, USA

Dr. Siddharth Kulkarni, The Hormiga Lab, The George Washington University, Washington,

D.C., USA

Dr. Tomas Ditrich, Faculty of Education, University of South Bohemia in Ceske

Budejovice, Czech Republic

Dr. Mihaly Foldvari, Natural History Museum, University of Oslo, Norway

Dr. V.P. Uniyal, Wildlife Institute of India, Dehradun, Uttarakhand 248001, India

Dr. John T.D. Caleb, Zoological Survey of India, Kolkata, West Bengal, India

Dr. Priyadarsanan Dharma Rajan, Ashoka Trust for Research in Ecology and the Environment (ATREE), Royal Enclave, Bangalore, Karnataka, India

\section{Fishes}

Dr. Neelesh Dahanukar, IISER, Pune, Maharashtra, India

Dr. Topiltzin Contreras MacBeath, Universidad Autónoma del estado de Morelos, México

Dr. Heok Hee Ng, National University of Singapore, Science Drive, Singapore

Dr. Rajeev Raghavan, St. Albert's College, Kochi, Kerala, India

Dr. Robert D. Sluka, Chiltern Gateway Project, A Rocha UK, Southall, Middlesex, UK

Dr. E. Vivekanandan, Central Marine Fisheries Research Institute, Chennai, India

Dr. Davor Zanella, University of Zagreb, Zagreb, Croatia

Dr. A. Biju Kumar, University of Kerala, Thiruvananthapuram, Kerala, India

Dr. Akhilesh K.V., ICAR-Central Marine Fisheries Research Institute, Mumbai Research

Centre, Mumbai, Maharashtra, India

Dr. J.A. Johnson, Wildlife Institute of India, Dehradun, Uttarakhand, India

\section{Amphibians}

Dr. Sushil K. Dutta, Indian Institute of Science, Bengaluru, Karnataka, India

Dr. Annemarie Ohler, Muséum national d'Histoire naturelle, Paris, France

\section{Reptiles}

Dr. Gernot Vogel, Heidelberg, Germany

Dr. Raju Vyas, Vadodara, Gujarat, India

Dr. Pritpal S. Soorae, Environment Agency, Abu Dubai, UAE.

Prof. Dr. Wayne J. Fuller, Near East University, Mersin, Turkey

Prof. Chandrashekher U. Rivonker, Goa University, Taleigao Plateau, Goa. India

Dr. S.R. Ganesh, Chennai Snake Park, Chennai, Tamil Nadu, India

Dr. Himansu Sekhar Das, Terrestrial \& Marine Biodiversity, Abu Dhabi, UAE
Birds

Dr. Hem Sagar Baral, Charles Sturt University, NSW Australia

Dr. Chris Bowden, Royal Society for the Protection of Birds, Sandy, UK

Dr. Priya Davidar, Pondicherry University, Kalapet, Puducherry, India

Dr. J.W. Duckworth, IUCN SSC, Bath, UK

Dr. Rajah Jayapal, SACON, Coimbatore, Tamil Nadu, India

Dr. Rajiv S. Kalsi, M.L.N. College, Yamuna Nagar, Haryana, India

Dr. V. Santharam, Rishi Valley Education Centre, Chittoor Dt., Andhra Pradesh, India

Dr. S. Balachandran, Bombay Natural History Society, Mumbai, India

Mr. J. Praveen, Bengaluru, India

Dr. C. Srinivasulu, Osmania University, Hyderabad, India

Dr. K.S. Gopi Sundar, International Crane Foundation, Baraboo, USA

Dr. Gombobaatar Sundev, Professor of Ornithology, Ulaanbaatar, Mongolia

Prof. Reuven Yosef, International Birding \& Research Centre, Eilat, Israel

Dr. Taej Mundkur, Wetlands International, Wageningen, The Netherlands

Dr. Carol Inskipp, Bishop Auckland Co., Durham, UK

Dr. Tim Inskipp, Bishop Auckland Co, Durham, UK

Dr. V. Gokula, National College, Tiruchirappalli, Tamil Nadu, India

Dr. Arkady Lelej, Russian Academy of Sciences, Vladivostok, Russia

Dr. Simon Dowell, Science Director, Chester Zoo, UK

Dr. Mário Gabriel Santiago dos Santos, Universidade de Trás-os-Montes e Alto Douro,

Quinta de Prados, Vila Real, Portugal

Dr. Grant Connette, Smithsonian Institution, Royal, VA, USA

Dr. M. Zafar-ul Islam, Prince Saud Al Faisal Wildlife Research Center, Taif, Saudi Arabia

Mammals

Dr. Giovanni Amori, CNR - Institute of Ecosystem Studies, Rome, Italy

Dr. Anwaruddin Chowdhury, Guwahati, India

Dr. David Mallon, Zoological Society of London, UK

Dr. Shomita Mukherjee, SACON, Coimbatore, Tamil Nadu, India

Dr. Angie Appel, Wild Cat Network, Germany

Dr. P.O. Nameer, Kerala Agricultural University, Thrissur, Kerala, India

Dr. Ian Redmond, UNEP Convention on Migratory Species, Lansdown, UK

Dr. Heidi S. Riddle, Riddle's Elephant and Wildlife Sanctuary, Arkansas, USA

Dr. Karin Schwartz, George Mason University, Fairfax, Virginia.

Dr. Lala A.K. Singh, Bhubaneswar, Orissa, India

Dr. Mewa Singh, Mysore University, Mysore, India

Dr. Paul Racey, University of Exeter, Devon, UK

Dr. Honnavalli N. Kumara, SACON, Anaikatty P.O., Coimbatore, Tamil Nadu, India

Dr. Nishith Dharaiya, HNG University, Patan, Gujarat, India

Dr. Spartaco Gippoliti, Socio Onorario Società Italiana per la Storia della Fauna "Giuseppe

Altobello", Rome, Italy

Dr. Justus Joshua, Green Future Foundation, Tiruchirapalli, Tamil Nadu, India

Dr. H. Raghuram, The American College, Madurai, Tamil Nadu, India

Dr. Paul Bates, Harison Institute, Kent, UK

Dr. Jim Sanderson, Small Wild Cat Conservation Foundation, Hartford, USA

Dr. Dan Challender, University of Kent, Canterbury, UK

Dr. David Mallon, Manchester Metropolitan University, Derbyshire, UK

Dr. Brian L. Cypher, California State University-Stanislaus, Bakersfield, CA

Dr. S.S. Talmale, Zoological Survey of India, Pune, Maharashtra, India

Prof. Karan Bahadur Shah, Budhanilakantha Municipality, Kathmandu, Nepal

Dr. Susan Cheyne, Borneo Nature Foundation International, Palangkaraja, Indonesia

Dr. Hemanta Kafley, Wildlife Sciences, Tarleton State University, Texas, USA

\section{Other Disciplines}

Dr. Aniruddha Belsare, Columbia MO 65203, USA (Veterinary)

Dr. Mandar S. Paingankar, University of Pune, Pune, Maharashtra, India (Molecular)

Dr. Jack Tordoff, Critical Ecosystem Partnership Fund, Arlington, USA (Communities)

Dr. Ulrike Streicher, University of Oregon, Eugene, USA (Veterinary)

Dr. Hari Balasubramanian, EcoAdvisors, Nova Scotia, Canada (Communities)

Dr. Rayanna Hellem Santos Bezerra, Universidade Federal de Sergipe, São Cristóvão, Brazil

Dr. Jamie R. Wood, Landcare Research, Canterbury, New Zealand

Dr. Wendy Collinson-Jonker, Endangered Wildlife Trust, Gauteng, South Africa

Dr. Rajeshkumar G. Jani, Anand Agricultural University, Anand, Gujarat, India

Dr. O.N. Tiwari, Senior Scientist, ICAR-Indian Agricultural Research Institute (IARI), New

Delhi, India

Dr. L.D. Singla, Guru Angad Dev Veterinary and Animal Sciences University, Ludhiana, India

Dr. Rupika S. Rajakaruna, University of Peradeniya, Peradeniya, Sri Lanka

Dr. Bahar Baviskar, Wild-CER, Nagpur, Maharashtra 440013, India

Reviewers 2018-2020

Due to pausity of space, the list of reviewers for 2018-2020 is available online.
The opinions expressed by the authors do not reflect the views of the Journal of Threatened Taxa, Wildlife Information Liaison Development Society, Zoo Outreach Organization, or any of the partners. The journal, the publisher, the host, and the partners are not responsible for the accuracy of the political boundaries shown in the maps by the authors.

\footnotetext{
Print copies of the Journal are available at cost. Write to:

The Managing Editor, JoTT,

c/o Wildlife Information Liaison Development Society,

No. 12, Thiruvannamalai Nagar, Saravanampatti - Kalapatti Road,

Saravanampatti, Coimbatore, Tamil Nadu 641035, India

ravi@threatenedtaxa.org
} 


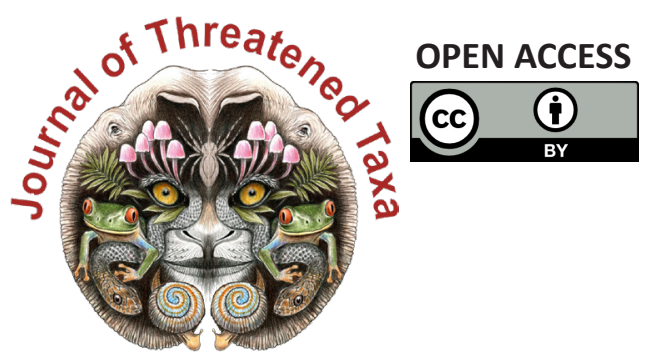

www.threatenedtaxa.org

The Journal of Threatened Taxa (JoTT) is dedicated to building evidence for conservation globally by publishing peer-reviewed articles online every month at a reasonably rapid rate at www.threatenedtaxa.org. All articles published in JoTT are registered under Creative Commons Attribution 4.0 International License unless otherwise mentioned. JoTT allows allows unrestricted use, reproduction, and distribution of articles in any medium by providing adequate credit to the author(s) and the source of publication.

\section{ISSN $0974-7907$ (Online) | ISSN $0974-7893$ (Print)}

\section{October 2021 | Vol. 13 | No. 12 | Pages: 19675-19886 \\ Date of Publication: 26 October 2021 (Online \& Print) DOI: 10.11609/jott.2021.13.12.19675-19886}

Articles

Roosting habits and habitats of the Indian Flying Fox Pteropus medius Temminck, 1825 in the northern districts of Tamil Nadu, India

- M. Pandian \& S. Suresh, Pp. 19675-19688

Diversity and distribution of avifauna at Warathenna-Hakkinda Environmental Protection Area in Kandy, Sri Lanka

- Dinelka Thilakarathne, Tithira Lakkana, Gayan Hirimuthugoda, Chaminda Wijesundara \& Shalika Kumburegama, Pp. 19689-19701

Grass species composition in tropical forest of southern India

- M. Ashokkumar, S. Swaminathan \& R. Nagarajan, Pp. 19702-19713

\section{Communications}

Habitat use and conservation threats to Wild Water Buffalo Bubalus arnee (Mammalia: Artiodactyla: Bovidae) in Koshi Tappu Wildlife Reserve, Nepal

- Reeta Khulal, Bijaya Neupane, Bijaya Dhami, Siddhartha Regmi, Ganesh Prasad Tiwari \& Manita Parajuli, Pp. 19714-19724

Get my head around owls: people perception and knowledge about owls of Andaman Islands

- Shanmugavel Sureshmarimuthu, Santhanakrishnan Babu, Nagaraj Rajeshkumar \& Honnavalli Nagaraj Kumara, Pp. 19725-19732

Abundance and diversity of threatened birds in Nangal Wetland, Punjab, India - Rajwinder Kaur \& Onkar Singh Brraich, Pp. 19733-19742

Evaluation of fish diversity and abundance in the Kabul River with comparisons between reaches above and below Kabul City, Afghanistan

- Ugyen Kelzang, Ahmad Farid Habibi \& Ryan J. Thoni, Pp. 19743-19752

New record of Myrmarachne melanocephala MacLeay, 1839 (Araneae: Salticidae) from Jharkhand, India and biogeographical implications of the co-occurrence of its ant model Tetraponera rufonigra Jerdon, 1851

- Rahul Kumar, Mirtunjay Sharma \& Ajay Kumar Sharma, Pp. 19753-19761

Diversity of spiders (Arachnida: Araneae) and the impact of pruning in Indian sandalwood plantations from Karnataka, India

-S. Padma 1 \& R. Sundararaj, Pp. 19762-19772

New records of cheilostome Bryozoa from the eastern coast of India encrusting on the exoskeleton of live horseshoe crabs of Indian Sundarbans

- Swati Das, Maria Susan Sanjay, Basudev Tripathy, C. Venkatraman \& K.A. Subramanian, Pp. 19773-19780

On the pteridophytes of Bherjan-Borajan-Padumoni Wildlife Sanctuary, Assam, India - Pranjal Borah \& Jayanta Barukial, Pp. 19781-19790

Population status of Heritiera fomes Buch.-Ham., a threatened species from Mahanadi Mangrove Wetland, India

- Sudam Charan Sahu, Manas Ranjan Mohanta \& N.H. Ravindranath, Pp. 19791-19798

Additions to the lichenized and lichenicolous fungi of Jammu \& Kashmir from Kishtwar High Altitude National Park

- Vishal Kumar, Yash Pal Sharma, Siljo Joseph, Roshinikumar Ngangom \& Sanjeeva Nayaka, Pp. 19799-19807

\section{Short Communications}

Is release of rehabilitated wildlife with embedded lead ammunition advisable? Plumbism in a Jaguar Panthera Onca (Mammalia: Carnivora: Felidae), survivor of gunshot wounds - Eduardo A. Díaz, Carolina Sáenz, E. Santiago Jiménez, David A. Egas \& Kelly Swing, Pp. 19808-19812

New record of the Sewing Needle Zipper Loach Paracanthocobitis linypha Singer \& Page, 2015 (Teleostei: Cypriniformes: Nemacheilidae) from the Chindwin drainage of Manipur, India

- Yumnam Rameshori, Yengkhom Chinglemba \& Waikhom Vishwanath, Pp. 19813-19817

Field identification characters to diagnose Microhyla mukhlesuri from closely related M. mymensinghensis (Amphibia: Microhylidae) and range extension of $M$. mukhlesuri up to West Bengal State, India

- Suman Pratihar \& Kaushik Deuti, Pp. 19818-19823
First report of Scipinia horrida (Stål) (Heteroptera: Reduviidae) from Assam, with comments on related genus Irantha Stål

- Anjana Singha Naorem, Santana Saikia, Anandita Buragohain, Rubina Azmeera Begum, Swapnil S. Boyane \& Hemant V. Ghate, Pp. 19824-19830

Flesh fly (Diptera: Sarcophagidae): male terminalia, diversity and expanded geographical distribution from India

- Kanholi Sreejith, Shuvra Kanti Sinha, Santanu Mahato \& Edamana Pushpalatha, Pp. 1983119836

Checklist of moths (Heterocera) of Tadong, Sikkim, India

- Prayash Chettri, Yuki Matsui, Hideshi Naka \& Archana Tiwari, Pp. 19837-19848

New distribution records of Begonia L., B. murina Craib and B. poilanei Kiew (Begoniaceae: Cucurbitales) for Laos

- Phongphayboun Phonepaseuth, Phetlasy Souladeth, Soulivanh Lanorsavanh, Shuichiro Tagane, Thyraphon Vongthavone \& Keooudone Souvannakhoummane Pp. 19849-19854

Notes

A recent sighting of the Stripe-backed Weasel Mustela strigidorsa (Mammalia: Carnivora: Mustelidae) in Hkakabo Razi Landscape, Myanmar

- Sai Sein Lin Oo, Tun Tun, Kyaw Myo Naing \& Paul Jeremy James Bates, Pp. 19855-19859

Are the uplifted reef beds in North Andaman letting nesting Olive Ridley Sea Turtle Lepidochelys olivacea stranded?

- Nehru Prabakaran, Anoop Raj Singh \& Vedagiri Thirumurugan, Pp. 19860-19863

First record of the orb-weaving spider Araneus tubabdominus Zhu \& Zhang, 1993 (Araneae: Araneidae) from India

- Souvik Sen, John T.D. Caleb \& Shelley Acharya, Pp. 19864-19866

The genus Catapiestus Perty, 1831 (Coleoptera: Tenebrionidae: Cnodalonini) from Arunachal Pradesh with one new record to India

- V.D. Hegde \& Sarita Yadav, Pp. 19867-19869

Rediscovery and extended distribution of Indigofera santapaui Sanjappa (Leguminosae: Papilionoideae) from the states of Maharashtra and Gujarat, India

- Kumar Vinod Chhotupuri Gosavi, Sanjay Gajanan Auti, Sharad Suresh Kambale \& Munivenkatappa Sanjappa, Pp. 19870-19873

Additional distribution records of Ceropegia anjanerica, an endemic and 'Endangered' lantern flower of the northern Western Ghats, India

- Samir Shrikant Maity, Ajay Natha Gangurde, Sharad Suresh Kambale, Avinash Ramchandra Gholave, Avinash Asraji Adsul, Ganesh Babaso Pawar \& Kumar Vinod Chhotupuri Gosavi, Pp. 19874-19877

Notes on the extended distribution of Impatiens megamalayana, a recently described balsam in Western Ghats, India

- Anoop P. Balan \& A.J. Robi, Pp. 19878-19883

Book Review

A look over on the scented tree of India (Santalum album - S. Suresh Ramanan \& A. Arunachalam, Pp. 19884-19886
Publisher \& Host
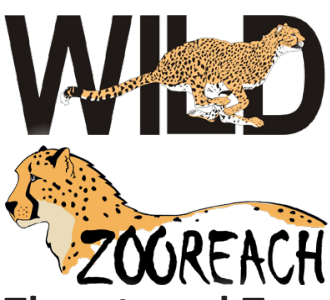

Threatened Taxa 